\title{
IRAS AND DISTANT GALAXIES
}

\author{
RYOHEI, KAWABE \\ Nobeyama Radio Observatory, National Astronomical Observatory
}

\begin{abstract}
Recent millimeter-wave observations of CO from the protogalaxy candidate IRAS F10214+4724 with single dishes and interferometers indicated that the huge amount of molecular gas is associated with the high-z galaxy with huge infrared luminosity. An overview of the results of recent observations of IRAS F10214 +4724 is shown. The implications of the results based on aperture synthesis $\mathrm{CO}$ observations and origin of the huge infrared luminosity (starburst or dust- enshrouded QSO) are discussed by comparing them with the "nearby" ultraluminous IRAS Galaxies.
\end{abstract}

\section{INTRODUCTION}

The formation and evolution of galaxies and QSOs, in terms of star formation history is very important in astronomy. The discoveries and detailed studies of ultraluminous IR galaxies in the local universe provided us several important keys for the investigation of the evolution of galaxies and QSOs such as: 1) ultraluminous galaxies as merger remnants, 2) ultraluminous galaxies as possible QSO progenitors, and 3) connection (link) between starbursts and AGN/QSOs (e.g., Sanders 1991; Kormendy and Sanders 1992). Two recent discoveries are very surprising; one is the discovery of a protogalaxy candidate, IRAS F10214+4742 at $\mathrm{z}=2.3$ and the other is the discovery of a candidate Zeldovich Pancake at $\mathrm{z}=3.4$ (Uson, Bagri, and Cornwell 1991; this detection of HI at $\mathrm{z}=3.4$ was not confirmed by the WSRT observations;Wieringa 1993).

IRAS F10214+4724 found by Rowan-Robinson et al.(1991) through an IRAS faint source survey, has a huge IR luminosity, $\mathrm{L}_{I R}=0.8 \times 10^{14} h^{-2}$ $L_{\odot}$ (rest frame luminosity estimated assuming $\mathrm{H}_{o}=100 \mathrm{hm} \mathrm{s}^{-1} \mathrm{Mpc}^{-1}$ and $\mathrm{q}_{\circ}$ $=0.5)$. This is ten times more luminous than the most luminous IRAS galaxy previously known, the Seyfert 2 cD galaxy IRAS $09014+4109\left(6 \times 10^{12} h^{-2}\right.$ $L_{\odot}$; Kleinmann et al. 1988). This huge IR luminosity was interpreted as being due to thermal emission from dust heated by AGN or newly formed stars in this galaxy, that is, a protogalaxy undergoing initial starburst or a radio quiet quasar heavily enshrouded in dust. The optical image consists of an unresolved source and a diffuse asymmetric source extended over 4 arcsec. Near-infrared images and the $\mathrm{H} \alpha$ line were taken by Soifer et al.(1991). They found an unresolved near-IR source and a very strong $\mathrm{H} \alpha$ line, but there was no evidence for a broad line region. Recently, the infrared image was resolved into two objects separated by 1 arcsec. and aligned north-south (Soifer et al. 1992). The $\mathrm{H} \alpha$ emission is centered on the southern component. 


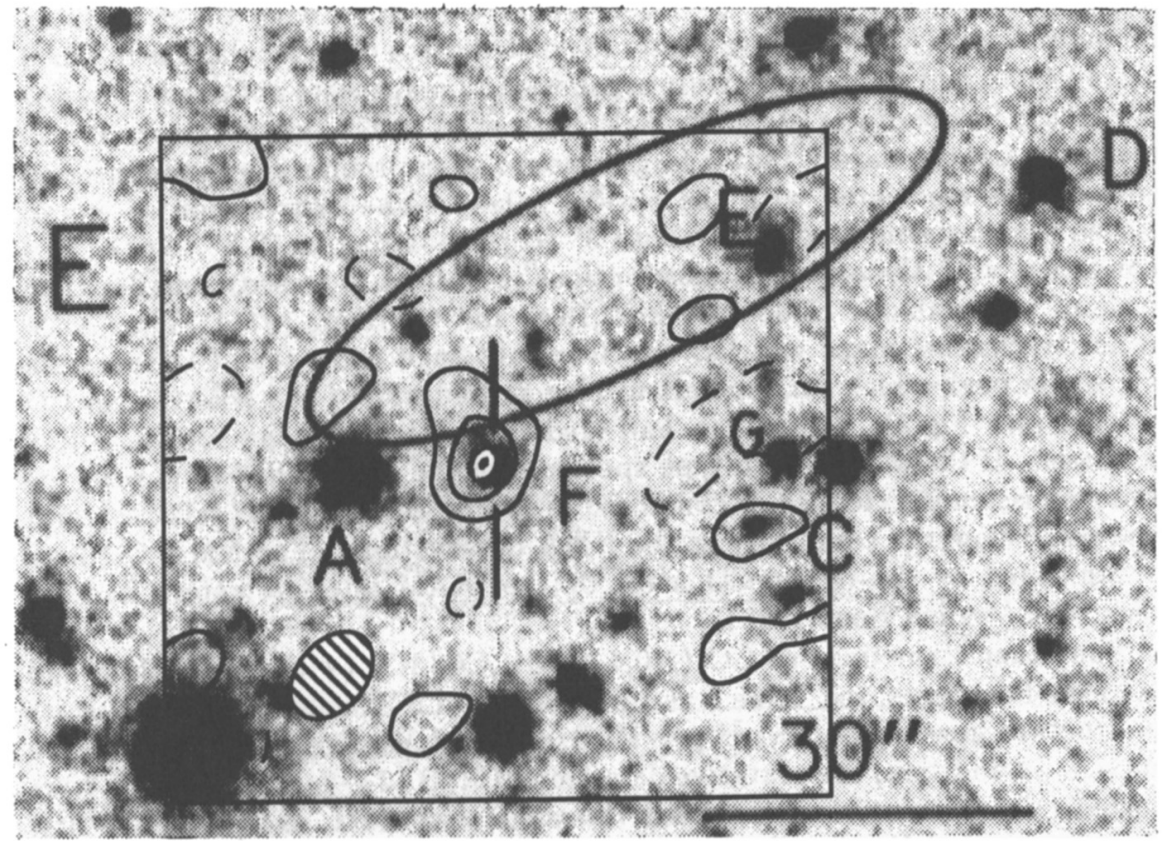

FIGURE I NMA CO(3-2) map (Kawabe et al. 1992) superimposed on the optical image of IRAS F10214+4724 (object F; Rowan Robinson et al. 1991). The total on-source integration time of $\mathrm{CO}$ observations was 31.7 hours. The synthesized beam was $8 . " 9 \times 6 . " 0\left(\mathrm{PA}=132^{\circ}\right)$. The three positions, $\mathrm{CO}$ optical, and VLA radio are coincident within 1 arcsec. The ellipse denotes the IRAS error of location of IRAS F10214+4724.

\section{MOLECULAR LINE AND SUB-MM OBSERVATIONS OF IRAS F10214+4724}

Brown and Vanden Bont (1991) reported the detection of CO(3-2) emission from IRAS F10214+4724. Observed CO emission (rest frequency $345.7960 \mathrm{GHz}$ ) was redshifted to around $105.2 \mathrm{GHz}(z=2.286)$. The similarity between the redshift of $\mathrm{CO}$ emission of 2.2867 and that of the optical galaxy implies that a huge amount of molecular gas is associated with the optical galaxy and that metal abundance in the galaxy is enriched through enough stellar cycle (e.g., $\mathrm{H}_{2}$ mass was estimated to be $(2-6) \times 10^{11} h^{-2} M_{\odot}$ by Solomon, Radford, and Downes 1992). Because of low spatial resolution of the IRAS observations, it was not clear whether the optical galaxy is actually associated with the infrared source or not, although there is indirect evidence for that the optical galaxy is the infrared source (e.g., a VLA source was found to be associated with the galaxy). How is metal enriched gas distributed? Dose it extended beyond the galactic disk scale (now collapsing protogalaxy), or is it concentrated to the optical galaxy? It would be natural for the gas to be associated with the galaxy because metal is produced by the stellar cycle. What is the energy source? This is another important question related to "nearby" ultraluminous galaxies. 
Apertnre synthesis $\mathrm{CO}(3-2)$ observations were done with the Nobeyama Millimeter Array (NMA) to investigate the distribution of molecular gas in IRAS F10214+4724, and the first aperture synthesis CO maps were obtained (Kawabe et al., 1992; Sakamoto et al. 1992; Sakamoto et al. 1993 this volume). It was revealed straightforwardly that a large amount of molecular gas (huge infrared luminosity) is associated with the optical galaxy. The distribution of metalenriched gas was estimated to be about 3" (corresponding to $12 \mathrm{~h}^{-1} \mathrm{kpc}$ ). This is consistent with the extended distributions in optical, 4 arcsec. (smaller than a scale of protogalactic cloud, $100 \mathrm{kpc}$;Rees and Ostriker, 1977). The $\mathrm{CO}(3-$ 2) velocity channel maps and $\mathrm{CO}$ spectrum obtained show that $\mathrm{CO}$ spectrum has a single peak which corresponds to blue-shifted half of the NRAO $12 \mathrm{~m} \mathrm{CO}$ spectrum (see Fig.2). More than half (red-shifted half) of the total CO flux was missed. If the double-horn type spectrum of the $12 \mathrm{~m} \mathrm{CO} \mathrm{(3-2)} \mathrm{is} \mathrm{real,} \mathrm{the}$ missing emission is extended or composed of several components (galaxies) with brightness below the sensitivity of the NMA observations. The NMA CO map was confirmed by the recent CO maps by the IRAM interferometer (Radford Brown, and Vanden Bout 1993).

The other transitions of CO (1-0,4-3,6-5), CO(3-2) (with IRAM 30m and NRO 45m Telescopes; see Table 1 and Figure 2) and [CI] emission (ground-state fine-structure lines ${ }^{3} \mathrm{P}_{2}-{ }^{3} \mathrm{P}_{1},{ }^{3} \mathrm{P}_{1}-{ }^{3} \mathrm{P}_{0}$;Brown and Vanden Bout 1992), were observed. In addition, the tentative detection of $\mathrm{H}_{2} \mathrm{O}\left(2_{11}-2_{02}\right)$ at $752 \mathrm{GHz}$ were reported (Encrenaz et al. 1993). All the single dish data, except for the first one by Brown and Vanden Bout, show single peak CO spectra which is nearly identical to the NMA CO spectrum. An "aperture photometry" analysis with different beam (aperture) sizes suggested that $\mathrm{CO}$ is distributed spatially over 10"-20" (Tsuboi and Nakai 1992).

Measurements of millimeter and submillimeter $(1.2 \mathrm{~mm}, 450,800 \mu \mathrm{m}$; $1.2 \mathrm{~mm}=370 \mu \mathrm{m}$ at rest frame) continua were done using IRAM $30 \mathrm{~m}$ and JCMT 15m telescopes (Downes et al. 1992; Clements et al. 1992; see Table 3 and Fig.3). The gas to warm dust ratio was estimasted to be 500, which is close to normal, and $T_{d u s t}$ was $80 \mathrm{~K}$. In IRAS F10214+4224, most heavy elements had been produced at near present-day abundances. This is not surprising because high-z $(\mathrm{z} \sim 4)$ QSOs shows their broad lines have an order of solar metallicity (Hamann and Ferland 1992). This indicates an earlier phase of metal enrichment.

\section{PHYSICAL NATURES OF IRAS F10214+4724}

The $\mathrm{H}_{2}$ mass was estimated by several authors and by several methods (Brown and Vanden Bout 1991; Solomon, Radford, and Downes et al. 1992 ; Kawabe et al. 1992 ; Sakamoto et al. 1992; Solomon, Downes, and Radford 1993) as,

$$
\mathrm{M}\left(\mathrm{H}_{2}\right) \sim(1-10) \times 10^{11} \mathrm{~h}^{-2} M_{\odot}
$$

The estimated $\mathrm{H}_{2}$ mass is comparable to the total mass of our Galaxy and several times larger than that of the most gas rich ultraluminous infrared galaxies (Table 2). The $\mathrm{H}_{2}$ surface density of IRAS F10214+4724 is comparable to that of ultraluminous infrared galaxies. The ratio of $\mathrm{H}_{2}$ gas to dynamical masses is roughly estimated as (Sakamoto et al. 1992), 


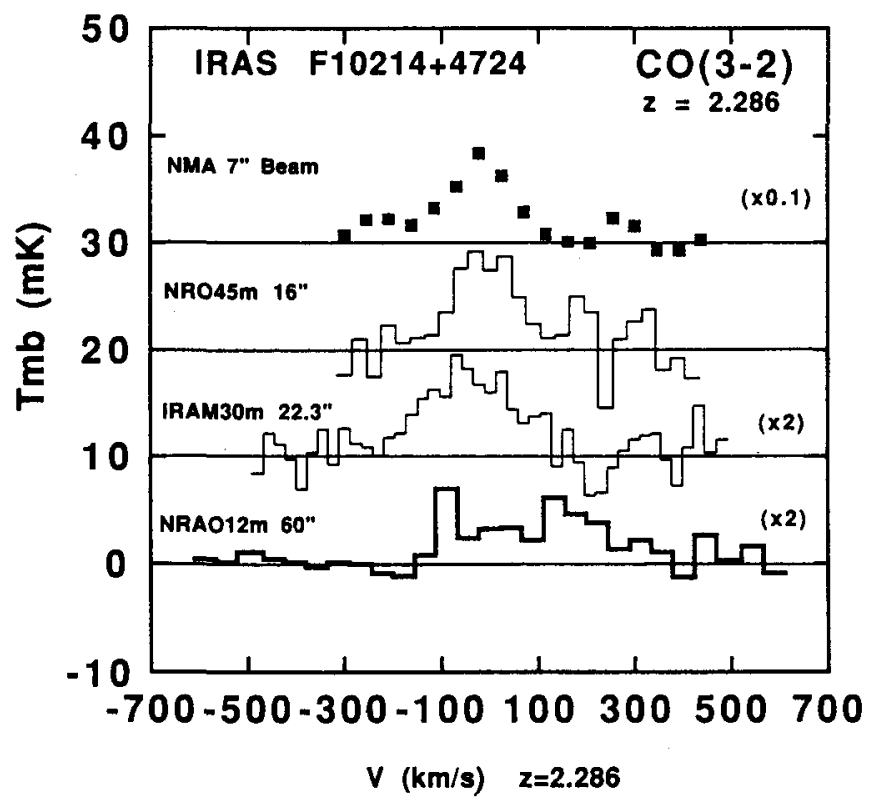

FIGURE II CO(3-2) spectra of IRAS F10214+4724 taken with different spatial resolutions; NMA - Sakamoto et al. 1992, NRO 45m - Tuboi and Nakai 1993, IRAM 30m - Solomon, Downes, and Radford 1993, NRAO 12m - Brown and Vanden Bout 1991).

$$
\mathrm{M}\left(\mathrm{H}_{2}\right) / \mathrm{M}_{\text {dynamical }} \sim(7-70) \times \sin ^{-2} i \text {. }
$$

This indicates that the galaxy is nearly face-on, unless the molecular gas mass is severely overestimated, and that in IRAS F10214+4724, a large fraction of mass would be in the form of molecular gas at $\mathrm{z}=2.3$ (20\% of present age of the universe). In this sense, IRAS F10214+4724 would be a young galaxy just in or (just after) the epoch of initial starburst. The process of chemical evolution of such galaxies as IRAS F10214+4724 is an important problem which should be addressed, together with primodial star formation in protogalaxies, to estimate the age of such galaxies.

The gas was found to be concentrated to the central $10 \mathrm{kpc}$ region of the galaxy (Kawabe et al. 1992; Sakamoto et al. 1993; Radford et al. 1993). There is no direct evidence for the extended or extra components of $\mathrm{CO}$ emission (CO fluxes, except for that by NRAO $12 \mathrm{~m}$ telescope, are roughly consistent with the point source model), although there is some indirect evidence. We have to be careful about "aperture photometry" of CO intensities with different beams of different telescopes, because flux scales are more uncertain between telescopes. Discussions of missing flux and comparison of spectral shapes are highly dependent on whether the double horn $\mathrm{CO}$ spectrum firstly and only obtained by Brown and Vanden Bout (1991) is real or not (this has not been confirmed). 


\section{ORIGIN OF FAR-IR LUMINOSITY}

What is origin of the huge IR luminosity, starburst or AGN in IRAS F 10214+4724? Here, I treat the following time scale consideration; gas consumption and the dynamical time scale of molecular gas.

Table 1. CO Observations of IRAS F10214+4724

\begin{tabular}{ccccccc}
\hline Line & Telescop & Beam Size & $\begin{array}{c}T_{\mathrm{mb}} \\
\text { (peak.mk) }\end{array}$ & $\begin{array}{c}\text { CO Flux } \\
(\mathrm{Jy} \cdot \mathrm{km} / \mathrm{s})\end{array}$ & $\begin{array}{c}\text { CO Velocity Extent } \\
(\mathrm{km} / \mathrm{s} . z=2.286)\end{array}$ & Ref. \\
\hline CO(1-0) & NRO45m & $48^{\prime \prime}$ & $4.5(3 \sigma)$ & $2.9 \pm 0.5$ & & 1 \\
\hline & NMA & $7^{\prime \prime}$ & 84 & $7.5 \pm 1.9$ & $-150 \sim+100$ & 2,3 \\
& NRO45m & $16^{\prime \prime}$ & 9.1 & $4.4 \pm 0.7$ & $-150 \sim+150$ & 1 \\
CO(3-2) & IRAM30m & $22.3^{\prime \prime}$ & 5.5 & $4.1 \pm 0.9$ & $-200 \sim+120$ & 4 \\
& IRAM30m & $24^{\prime \prime}$ & 4.6 & 10.4 & $-250 \sim+70$ & 5 \\
& NRAO12m & $60^{\prime \prime}$ & 3.6 & 26 & $-170 \sim+250$ & 6 \\
\hline CO(4-3) & IRAM30m & $18^{\prime \prime}$ & 5.5 & 15.5 & $-180 \sim+100$ & 5 \\
\hline CO(6-5) & IRAM30m & $13.6^{\prime \prime}$ & 3.6 & $9.4 \pm 2.0$ & $-200 \sim+120$ & 4 \\
\hline
\end{tabular}

NOTE: 1. Tsuboi and Nakai (1993), 2. Kawabe et al. (1992), 3. Sakamoto et al. (1992), 4. Solomon, Downes, and Radford (1993), 5. Brown and Vanden Bout (1992), 6. Brown and Vanden Bout (1991).

Table 2.

IRAS F10214+4724 and Ultraluminous IRAS Galaxies / QSOs

\begin{tabular}{|c|c|c|c|}
\hline & IRAS F10214+4724 & $\begin{array}{c}\text { Ultraluminous Infrare } \\
\text { Cool Sample }\end{array}$ & $\begin{array}{l}\text { ies and Quasars } \\
\text { Warm Sample }\end{array}$ \\
\hline $\log \mathrm{L}_{\mathrm{IR}}\left(\mathrm{L}_{\odot}\right)$ & $13.9\left(+\log h^{-2}\right)$ & $11.93-12.51$ & $11.89-13.02$ \\
\hline $\log M\left(H_{2}\right)\left(M_{\odot}\right)$ & $11-12$ & $9.77-10.73$ & $9.77-10.87$ \\
\hline $\mathrm{LM}^{-1}\left(\mathrm{~L}_{\odot} \mathrm{M}_{\odot}{ }^{-1}\right)$ & $60-600$ & $\begin{array}{c}16-77 \\
44 \text { (average) }\end{array}$ & $\begin{array}{c}26-209 \\
72 \text { (average) }\end{array}$ \\
\hline 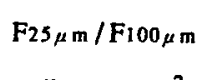 & $\begin{array}{c}0.3 \pm 0.1 \\
\text { (at rest frame) }\end{array}$ & $\begin{array}{c}0.05-0.38 \\
0.145 \text { (average) }\end{array}$ & $\begin{array}{c}0.2-0.86 \\
0.351 \text { (average) }\end{array}$ \\
\hline$\Sigma_{H 2}\left(M_{\odot} p^{-2}\right)$ & $>2-10 \times 10^{3}$ & $2 \times 10^{4}(\operatorname{Arp} 220)$ & $>0.95 \times 10^{4}($ Mrk231) \\
\hline References & $1,2,3,4$ & 5,6 & 5,6 \\
\hline
\end{tabular}

NOTE: The cool and warm samples of ultraluminous infrared galaxies and QSOs were taken from the lists by Sanders (1991); the cool sample consists of cooler 14 samples of the complete IRAS Bright Galaxies, and the warm sample consists of 11 samples of ultraluminous infrared galaxies and QSOs with $\mathrm{F} \nu$ $>1.5 \mathrm{Jy}$ and $\mathrm{F} \nu(25) / \mathrm{F} \nu(60)>0.2$ (E1821+643 is excluded because CO was not detected; Barvainis, Antonucci, and Coleman 1993). The $\mathrm{H}_{2}$ gas surface densities of typical galaxies of the two samples, Arp220 and Mrk231, are shown. 1. Rowan-Robinson et al. 1991, 2. Kawabe et al. (1992), 3. Sakamoto et al. (1992), 4. Clements et al. 1992, 5. Sanders et al. 1991, 6 Okumura et al. 1991. 


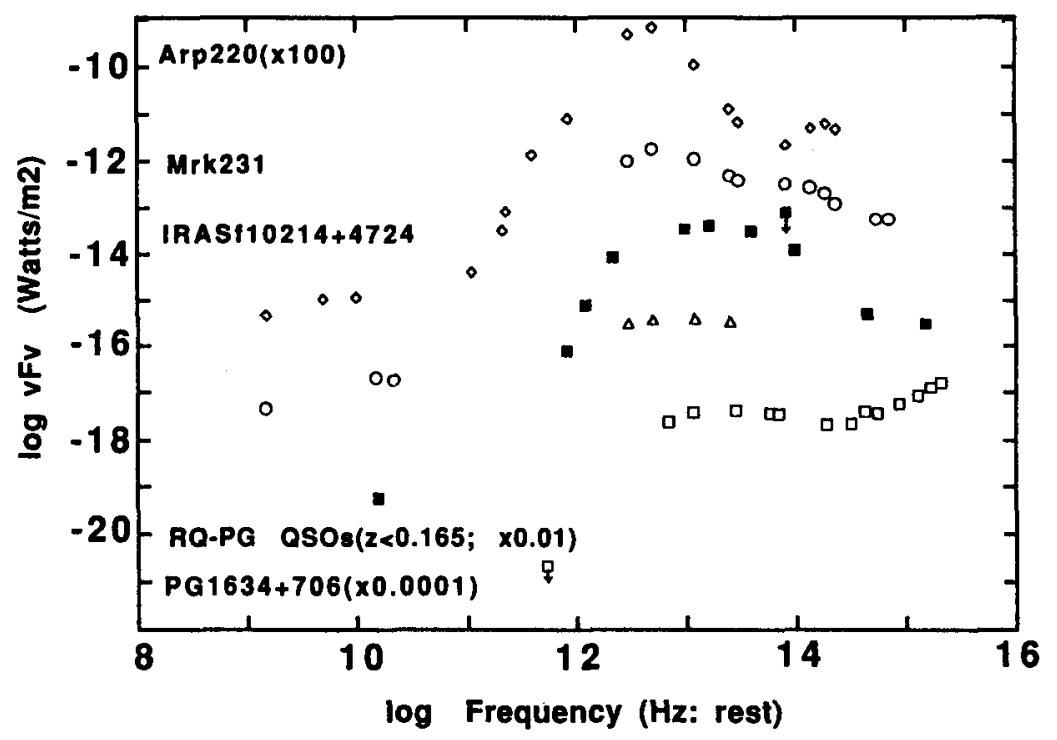

FIGURE III Spectral energy distributions of IRAS F10214+4724, typical ultraluminous IR galaxies Arp220 ("cool" sample) and Mrk231 ("warm" sample), and radio quiet $P G$ quasars (average of 12 nearby quasars at $z<0.165$ and PG1634+706 at $z=1.334$; Sanders et al. 1989). Data of IRAS F10214+4724 were taken from Rowan-Robinson et al.(1991), Clements et al.(1992), Dawnes, Solomon, and Radford (1993).

Molecular gas consumption time scales can be estimated for two cases, AGN and starburst origins. the gas consumption (accretion) rate for the AGN origin is $70 \mathrm{~h}^{-2} M_{\odot}$ year $^{-1}$, assuming a mass to radiant energy conversion efficiency rate of $10 \%$ in AGN, and assuming mainly molecular gas is consumed (i.e., $L=0.1 \dot{M} c^{2} ;$ Norman and Scoville 1988). The time scale is about $(0.15-0.4)$ $\times 10^{10}$ years (about one third of the Hubble time). The time scale in a case of starburst origin, is as short as $(1-3) \times 10^{7}$ years, assuming that the star formation rate is $1 M_{\odot}$ year ${ }^{-1}$ for $L_{I R}=10^{10} L_{\odot}$ (Young and Scoville 1983). Dynamical time scale of molecular gas is $\left(2 \pi r / V_{\text {rot }}\right) \sim(2-5) \times 10^{8} h^{-1}$ years, assuming that the molecular gas forms a disk, and the rotational velocity is 200 $\mathrm{kms}^{-1} / \sin i\left(i=30-90^{\circ}\right)$ and the radius of rotation is $16 \mathrm{~h}^{-1} \mathrm{kpc}$.

Such a short time scale for the gas consumption in the starburst model suggests that gas is converted to stars very quickly, before the gas system collapses and forms a gas disk with a well-defined rotational axis through reconfiguration of angular momentum, and the huge IR luminosity would be generated in an instant (about a few tenths \% of the Hubble time). The merging of protogalactic clouds might trigger such a starburst, and the bulge of a disk galaxy or an elliptical galaxy may be formed from star formation in such a galaxy. However, the very short starburst duration might limit opportunity to observe such an object (Kawabe et al. 1992). The molecular gas actually forms a disk with the dynanical time scale; the starburst model with a much shorter time scale will be excluded. 
An existing large amount of metal-enriched molecular gas shows the formation of large number of stars before $z=2.286$. The duration of star formation is much longer compared with the starburst duraton estimated above, if we assume star formation started from $\mathrm{z}=5-10$ (corresponding to the duration from $\mathrm{z}=5-10$ to $\mathrm{z}=2.3 ; 10-15 \%$ of the Hubble time).

The Far IR spectrum also suggests AGN origin of the huge infrared luminosity because the spectrum has $12,25 \mu \mathrm{m}$ excess compared with the starburst galaxies. The spectrum is very similar to those of the warm ultraluminous galaxies (Fig.3), e.g., that of a Seyfert I galaxy (radio quiet quasar) Mrk231. The excess is more prominent in the case of UV-excess radio quiet quasers (Fig. 3 ). The $25 \mu \mathrm{m}$ to $100 \mu \mathrm{m}$ flux ratio of IRAS F $10214+4724$ at rest frame is estimated to be 0.3 (Clements et al 1992) and the averaged ratio over the warm sample is 0.351 (Table 2). All galaxies and quasars in the warm sample have Seyfert optical spectra and the mid-infrared excess in the sample and radio quiet quasers was interpreted as reprocessed emission from nuclear dust disks surrounding central active galactic nuclei (e.g., Sanders et al. 1989; Barvanis 1990). It seems that the excess cannot be explained by star formation and that the excess is due to the AGN heated warm/hot dust in circumnuclear disk, although the $60,100 \mu \mathrm{m}$ flux (at rest flame) might be explained by dust (of galaxy disk or envelope of nuclear dust disk) heated by newly-formed stars. The huge IR luminosity would be likely due to the combination of starburst (far-IR part at rest frame) and AGN (mid-IR).

IRAS detected several high-z radio quiet quasars and recent submillimeter observations detected a high-redshift BAL quasar (see Table 3 ) and high-redshift quasars (Andreani, Flanca, and Cristiani 1993). The detected emissions are thought to be thermal emission from dust in nuclei and disks of host galaxies. These high-z quasars should also be addressed as well as nearby ultraluminous IR galaxies in terms of the origin of IR luminosity and the link between starbursts and QSOs.

Table 3.

High Redshift IR Galaxies and Radio Quiet Quasars detected with IRAS

\begin{tabular}{|c|c|c|c|c|c|c|c|c|c|c|}
\hline \multirow[b]{2}{*}{ Name } & & \multicolumn{4}{|c|}{$F_{y}(m \mathrm{Jy})$} & \multirow[b]{2}{*}{$800_{\mu \mathrm{m}}$} & \multirow[b]{2}{*}{$1.2 / 1.3 \mathrm{~mm}$} & \multirow{2}{*}{ Ref } \\
\hline & $z$ & $\left(L_{\odot}\right)$ & $12 \mu \mathrm{m}$ & $25 \mu \mathrm{m}$ & $60 \mu \mathrm{m}$ & $100 \mu \mathrm{m}$ & $450 \mu \mathrm{m}$ & & & \\
\hline IRASF $10214+4724$ & 2.286 & 13.9 & $<90$ & $75 \pm 31$ & $230 \pm 60$ & $330 \pm 240$ & $370 \pm 100$ & $60 \pm 15$ & $9.6 \pm 1.4$ & $1,2,3$ \\
\hline K1413+117 & 2.546 & $>13$ & & & & & $224 \pm 38$ & $44 \pm 8$ & $<35(1.3 \mathrm{~mm})$ & 4 \\
\hline PG1008+113 & 1.28 & 13.6 & 130 & $178 \pm 56$ & $<140$ & $<315$ & & & & 5 \\
\hline PG $1148+549$ & 0.969 & 13.7 & $<75$ & 120 & 196 & 410 & & & & 5 \\
\hline PG1206+459 & 1.158 & 13.7 & $207 \pm 36$ & $<130$ & 250 & $<1000$ & & & & 5 \\
\hline PG1248+401 & 1.03 & 13.5 & $<117$ & $<200$ & $224 \pm 51$ & $<378$ & & & & 5 \\
\hline PG1338+416 & 1.29 & 13.8 & $113 \pm 33$ & 80 & $<140$ & $<347$ & & & & 5 \\
\hline PG1634+706 & 1.334 & 14.05 & $61 \pm 13$ & $147 \pm 14$ & $318 \pm 23$ & $343 \pm 72$ & & & $<4.2(1.3 \mathrm{~mm})$ & 5,6 \\
\hline
\end{tabular}

NOTE: 1. Rowan-Robinson et al. 1991, 2. Clements et al. (1992), 3. Downes, Radford, and Solomon (1993), 4. Barvainis, Antonucci, and Coleman 1992, 5. Sanders et al. 1989, 6 Chini et al. 1989. 


\section{CONCLUSIONS}

Conclusions are summarized in the followings.

1. The idea that the optical galaxy with a redshift of 2.286 is one of sources of the huge infrared luminosity has been made more plausible by the results of the aperture synthesis $\mathrm{CO}$ observations. No direct and clear evidence for the other components.

2. NMA (and also IRAM interferometer) results indicate that metalenriched molecular gas sufficiently collapsed at least to the galaxy disk scale.

3. At present, it is not clear whether the origin of the huge infrared luminosity in IRAS F10214+4724 is starburst or AGN, that is, whether IRAS F10214+4724 is a protogalaxy or a dust-enshrouded QSO. The gas consumption time scale consideration, the 12 and $25 \mu \mathrm{m}$ excess, large $\mathrm{L}_{I R} / \mathrm{M}\left(\mathrm{H}_{2}\right)$ ratio, and the detected [CIV] line favor the dust-enshrouded QSO model. For both of them, however, a large amount of molecular gas mainly is contributed to the energy production in a protogalaxy or a host galaxy of QSO. In addition, the obtained high gas surface density indicated that many stars will be formed even when a large amount of molecular gas is fed to a central engine (AGN). The detailed investigation of molecular gas in such high-z galaxies as IRAS F10214+4724 is very important as well as for relatively "nearby" ultraluminous IR galaxies.

4. It is very clear that huge millimeter arrays such LMA, MMA projects in the future would be powerful tools for the investigation of such distant galaxies such as IRAS F10214+4724 and proto-galaxy/QSOs (Loeb 1993).

\section{REFERENCES}

Andreani, P., Franca, F.L., and Cristiani, S., 1993, Mon.Not.R.Astron.Soc, in press.

Barvanis, R., 1990, Ap.J., 353, 419

Barvanis, R., Antonucci, R., and Coleman, P., 1992, Ap.J.(Letters), 399, L19

Brown, R.L., and Vanden Bout, P.A., 1992, A.J., 397, L11

Brown, R.L., and Vanden Bout, P.A., 1992, Ap.J.(Letters), 397, L11

Chini, R., Kreysa, E., and Beirmann, P.L., 1989, Astron.Astrophys., 219,87

Clements, D.L., Rowan-Robinson, M., Lawrence, A., Broadhust, T., and McMahon, R., 1992, Mon.Not.R.Astr.Soc., 256, 35

Downes, D., et al. 1992, Ap.J., in press.

Encrenaz, P.J., Combes, F., Casoli, F., Gerin, M., Pagani, L., Horellou, C., and Gac, C., 1993, Astron.Astrphys., in press.

Hamann, F. and Ferland, G., 1992, Ap.J.(Letters), 391, L53

Kawabe, R., Sakamoto, K., Ishizuki, S., and Ishiguro, M., 1992, Ap.J.(Letters), 397, L23

Kleinmann et al. 1988, Ap.J.(Letters), 397,L23

Kormendy, J., and Sanders, D.B., 1992, Ap.J.(Letters), 390, L53 
Loeb, A., 1993, Ap.J.(Letters), 404, L37

Norman C., Scoville, N.Z., 1988, Ap.J., 322, 124

Okumura S.K., Kawabe, R., Ishiguro, M., Kasuga, T., Morita, K.-I., and Ishizuki, S., 1991, in Dynamics of Galaxies and Their Molecular Cloud Distribution, ed. F. Combes and F. Casoli(Dordrecht:Kluwer), 425

Radoford, S.J.E., Brown, R., and Vanden Bout, P.A. 1993, Astron. Astrphys., in press,

Rees, M., and Ostriker, J.P., 1977, MNRAS, 179, 541

Rowan-Robinson, M. et al. , 1991, Nature, 351, 719.

Sakamoto, K., Ishizuki, S., Kawabe, R., and Ishiguro, M., 1992, Ap.J.(Letters), 397,L27

Sakamoto, K., Kawabe, R., Ishizuki, S., Ishiguro, M., 1993 this volume

Sanders, D.B., 1991, in Dynamics of Galaxies and Their Molecular Clond Distribution, ed. F. Combes and F. Casoli(Dordrecht:Kluwer), 417

Sanders, D.B., Phinny, E.S., Neugebaner, G., Soifer, B.T., and Matthews, K., 1989, Ap.J., 347, 29

Scoville, N.Z., Sanders, D.B., Sargent, A.I., Soifer, B.T., and Tinny, C.G., 1989, Ap.J.(Letters), 345, L25

Scoville, N.Z., Sargent, A.I., Sanders, D.B., and Soifer, B.T., 1991, Ap.J.(Letters), 366, L5

Scoville, N.Z., and Young, J., 1983, Ap.J., 265, 148

Soifer, B.T., Neugebaner, G., Matthews, K., and Lawrence, C., 1992, Ap.J., 399, L55

Soifer et al. 1991, Ap.J.(Letters), 318, L55.

Solomon, P.M., Radford, P.M., and Downes, D., 1992, Nature, 356, 318

Solomon, P.M., Downes, D., Radford, P.M., 1993, Ap.J., in press.

Tsuboi, M., and Nakai, N. 1993, P.A.S.J.(Letters), 44, No.6, L241.

Uson, J.M., Bagri, D.S., and Cornwell, T.J., 1991, Phys.Rev.Letters, vol. 69, No. 24,3328

Wieringa, M.H., 1993, private communication 


\section{DISCUSSION}

R. Ekers Comment: There is a well documented result showing that discovery measurements have a strong bias in which the effect is overestimated compared with the eventual more accurate measurements. This occurs because noise in the right direction will take a weak signal over a threshold for detection. Perhaps this can explain some of the discrepancy between the very exciting discovery result for $\mathrm{CO}$ in F10214 + 4724 compared with the confirmation observations.

M. Wieringa Comment: New more sensitive Westerbork observations by A.G. de Bruyn (NFRA) do not show evidence for the emission feature seen in the VLA observations by Uson et al (excluded at $7 \sigma$ ). The westerbork observation do confirm the absorption feature. 